\title{
Teen BD patients show specific gray matter deficits
}

Published online: 20 September 2012

(C) Springer Healthcare 2012

medwireNews: Results from a US study show that adolescents with bipolar I disorder (BD I) who have experienced their first episode of mania have gray matter deficits in the left subgenual cingulate cortex.

The findings, published in Bipolar Disorders, suggest that such deficits are present at the onset of mania and are not the result of multiple mood episodes or prolonged exposure to medication, says the team.

Manpreet Singh (Stanford University, California) and colleagues studied 26 adolescents, aged 13 to 18 years, with BD I who had experienced a first episode of mania in the previous 12 months and 24 mentally healthy adolescents (controls). There were no significant differences between the groups regarding age, gender, pubertal stage, socioeconomic status, race, or handedness.

All of the participants underwent magnetic resonance imaging, and whole-brain voxel-based morphometry was used to compare brain volumes between the groups.

The researchers found that BD I patients did not differ from controls in total brain volume (TBV), total gray matter volume, and total white matter volume.

However, after accounting for TBV, age, and IQ, the team found that BD I patients had significantly reduced gray matter volume in the left subgenual anterior cingulate cortice (Brodmann area $25 \mathrm{sg}$ ) compared with controls.

There were no significant between-group differences regarding gray or white matter volume in other brain regions, the researchers note.

Singh and colleagues conclude: "Our results indicate that, compared to demographically similar healthy controls, adolescents with BD I are characterized by a significant reduction in left subgenual anterior cingulate cortice volume.”

They add that the findings "suggest a neural basis for symptoms associated with BD that accounts for abnormal hedonic manifestations and impulsivity through disruption of healthy prefrontal cortical development."

By Mark Cowen, Senior medwireNews Reporter

\section{Reference}

Bipolar Disord 2012; 14: 585-596 